\title{
Author Correction: No evidence for a bilingual executive function advantage in the ABCD study
}

Anthony Steven Dick (D), Nelcida L. Garcia, Shannon M. Pruden, Wesley K. Thompson, Samuel W. Hawes, Matthew T. Sutherland (D, Michael C. Riedel, Angela R. Laird (D) and Raul Gonzalez

Correction to: Nature Human Behaviour https://doi.org/10.1038/s41562-019-0609-3, published online 5 August 2019.

In the Supplementary Information originally posted, Supplementary Table 2 should have aggregated the number of participants speaking European languages other than Spanish and non-European languages other than Asian languages. The error has been corrected online.

Published online: 25 September 2019

https://doi.org/10.1038/s41562-019-0756-6 